\title{
Uniqueness and Asymptotic Behavior of Positive Solutions for a Fractional-Order Integral Boundary Value Problem
}

\author{
Min Jia, Xin Liu, and Xuemai Gu \\ Communication Research Center, Harbin Institute of Technology, Harbin 150080, China \\ Correspondence should be addressed to Min Jia, jiamin@hit.edu.cn
}

Received 18 July 2012; Accepted 7 August 2012

Academic Editor: Xinguang Zhang

Copyright (C) 2012 Min Jia et al. This is an open access article distributed under the Creative Commons Attribution License, which permits unrestricted use, distribution, and reproduction in any medium, provided the original work is properly cited.

We study a model arising from porous media, electromagnetic, and signal processing of wireless communication system $-\Phi_{\mathrm{t}}{ }^{\alpha} x(t)=f\left(t, x(t), x^{\prime}(t), x^{\prime \prime}(t), \ldots, x^{(n-2)}(t)\right), 0<t<1, x(0)=x^{\prime}(0)=\cdots=$ $x^{(n-2)}(0)=0, x^{(n-2)}(1)=\int_{0}^{1} x^{(n-2)}(s) d A(s)$, where $n-1<\alpha \leq n, n \in \mathbb{N}$ and $n \geq 2, \Phi_{\mathbf{t}}{ }^{\alpha}$ is the standard Riemann-Liouville derivative, $\int_{0}^{1} x(s) d A(s)$ is linear functionals given by RiemannStieltjes integrals, $A$ is a function of bounded variation, and $d A$ can be a changing-sign measure. The existence, uniqueness, and asymptotic behavior of positive solutions to the singular nonlocal integral boundary value problem for fractional differential equation are obtained. Our analysis relies on Schauder's fixed-point theorem and upper and lower solution method.

\section{Introduction}

Recently, fractional-order models have proved to be more accurate than integer order models, that is, there are more degrees of freedom in the fractional-order models.Indeed, we can find numerous applications in viscoelasticity, electrochemistry control, porous media, electromagnetic, and signal processing of wireless communication system. Especially, in application of the digital signal processing, the fractional digital signal processing can greatly improve the high frequency components of signal, enhance an intermediate frequency component of signal, and reserve nonlinear low frequency signal. According to this analysis, fractional differential equation applied to the edge information extraction will get higher signal to noise ratio than that of the traditional method based on one- or-two order differential equation. 
Many applications on digital signal processing were found recently, for example, Hartley et al. [1] derived a closed-loop control electromagnetic and signal processing system of equations with the Chua resistor

$$
\begin{gathered}
\Phi_{\mathrm{t}}^{\alpha} x(t)=a \Phi_{\mathrm{t}}^{\alpha-1}(y(t)-x(t))-\frac{b}{7}\left(4 x(t)-x^{3}(t)\right), \\
\frac{d y(t)}{d t}=x(t)-y(t) .
\end{gathered}
$$

Caputo [2] presented a fractional model system to describe the relationship between the electric field $E$ and the current density $D$ in the study of a electrochemical polarization medium,

$$
\gamma \oplus_{\mathrm{t}}^{\nu} D(t)+\alpha D(t)=\sigma E(t)+\delta \Phi_{\mathrm{t}}^{\nu} E(t)
$$

where $\gamma, \alpha, \sigma, \delta$ are constants and $v$ is a real number. Anastasio [3] believed that signal processing of communication system of vestibule visual reflex effect is fractional, which can be described by the following model

$$
\tau_{1} r^{\prime}(t)+r(t)=\tau_{1} \tau_{2} \Phi_{\mathrm{t}}^{\alpha+1} v(t)+\tau_{1} \Phi_{\mathrm{t}}^{\alpha} v(t)
$$

where $r(t)$ is the vestibular reflex nerve discharge rate, $v(t)$ is the head of the rotational angular velocity, $\alpha$ is fraction.

Motivated by systems (1.1)-(1.3) and their application background in electromagnetic and signal processing of wireless communication system, in this paper, we consider the existence, uniqueness, and asymptotic behavior of positive solutions for the higher nonlocal fractional differential equation

$$
\begin{gathered}
-\Phi_{\mathfrak{t}}{ }^{\alpha} x(t)=f\left(t, x(t), x^{\prime}(t), x^{\prime \prime}(t), \ldots, x^{(n-2)}(t)\right), \quad 0<t<1, \\
x(0)=x^{\prime}(0)=\cdots=x^{(n-2)}(0)=0, \quad x^{(n-2)}(1)=\int_{0}^{1} x^{(n-2)}(s) d A(s),
\end{gathered}
$$

where $n-1<\alpha \leq n, n \in \mathbb{N}$ and $n \geq 2, \Phi_{\mathfrak{t}}{ }^{\alpha}$ is the standard Riemann-Liouville derivative, $\int_{0}^{1} x(s) d A(s)$ is linear functionals given by Riemann-Stieltjes integrals, $A$ is a function of bounded variation, and $d A$ can be a changing-sign measure, and $f:(0,1) \times(0,+\infty)^{n-1} \rightarrow$ $[0,+\infty)$ is continuous, $f$ may be singular at $x_{i}=0$ and $t=0,1$.

The nonlocal integral-boundary value problems represent a class of interesting and important problems arising in physical, biological, and chemical processes and have attracted the attention of Khan [4], Gallardo [5], Karakostas and Tsamatos [6], Ahmad et al. [7], Feng et al. [8], Corduneanu [9], and Agarwal and O'Regan [10]. 
When $\alpha$ is an integer, a lot of work has been done dealing with nonlocal 3-point boundary value problems, see [11, 12]. In [12], Eloe and Ahmad studied the following $n$ thorder nonlocal differential equation

$$
\begin{gathered}
u^{(n)}(t)+a(t) f(u)=0, \quad 0<t<1, \\
u(0)=u^{\prime}(0)=\cdots=u^{(n-2)}(0)=0, \quad u(1)=\eta u(\xi),
\end{gathered}
$$

where $0<\xi<1,0<\eta \xi^{n-1}<1$. By applying the fixed point theorem in cones, the authors prove the existence of at least one positive solution when $a$ and $f$ is continuous and $f$ is either sublinear or superlinear. Recently, Hao et al. [13] studied the existence of positive solutions for the BVP (1.5) with integer order $n$, and nonlinear term is replaced by $a(t) f(t, x(t))$, where $a$ can be singular at $t=0,1, f$ can be singular at $x=0$ and no singularity at $t=0,1$.

If $\alpha$ is fractional, Yuan [14] studied the $(n-1,1)$-type conjugate boundary value problem

$$
\begin{gathered}
\Phi_{\mathfrak{t}}^{\alpha} u(t)+f(t, u(t))=0, \quad 0<t<1, n-1<\alpha \leq n, n \geq 3, \\
u(0)=u^{\prime}(0)=\cdots=u^{(n-2)}(0)=0, \quad u(1)=0
\end{gathered}
$$

where $f$ is continuous and semipositone, $\boldsymbol{\Phi}_{\mathbf{t}}{ }^{\alpha}$ is the standard Riemann-Liouville derivative. By giving properties of Green's function and using the Guo-Krasnosel'skii fixed-point theorem on cones, the existence of multiple positive solutions were obtained. More recently, Zhang [15] considered the following BVP whose nonlinear term and boundary condition contain derivatives of unknown functions

$$
\begin{gathered}
\Phi_{\mathrm{t}}^{\alpha} x(t)+q(t) f\left(x, x^{\prime}, \ldots, x^{(n-2)}\right)=0, \quad 0<t<1, n-1<\alpha \leq n, \\
x(0)=x^{\prime}(0)=\cdots=x^{(n-2)}(0)=x^{(n-2)}(1)=0,
\end{gathered}
$$

where $\Phi_{t}{ }^{\alpha}$ is the standard Riemann-Liouville fractional derivative of order $\alpha, q$ may be singular at $t=0$ and $f$ may be singular at $x=0, x^{\prime}=0, \ldots, x^{(n-2)}=0$, by using fixed-point theorem of the mixed monotone operator, the unique existence result of positive solution to problem (1.7) was established. Other some recent results, see [16-20]

Motivated by the results mentioned above, in this paper, we study the existence, uniqueness, and asymptotic behavior of positive solutions for the BVP (1.4) where the nonlinear terms and boundary conditions all involve derivatives of unknown functions and with Riemann-Stieltjes integral boundary condition, moreover $f$ may be singular at $x_{i}=0$ and $t=0,1$. Our main tool relies on Schauder's fixed-point theorem and upper and lower solution method.

\section{Preliminaries and Lemmas}

In this section, we present here the necessary definitions from fractional calculus theory. These definitions can be found in the recent literatures. 
Definition 2.1 (see [21,22]). The Riemann-Liouville fractional integral of order $\alpha>0$ of a function $x:(0,+\infty) \rightarrow \mathbb{R}$ is given by the following:

$$
I^{\alpha} x(t)=\frac{1}{\Gamma(\alpha)} \int_{0}^{t}(t-s)^{\alpha-1} x(s) d s
$$

provided that the right-hand side is pointwise defined on $(0,+\infty)$.

Definition 2.2 (see [21, 22]). The Riemann-Liouville fractional derivative of order $\alpha>0$ of a function $x:(0,+\infty) \rightarrow \mathbb{R}$ is given by the following:

$$
\Phi_{\mathfrak{t}}^{\alpha} x(t)=\frac{1}{\Gamma(n-\alpha)}\left(\frac{d}{d t}\right)^{n} \int_{0}^{t}(t-s)^{n-\alpha-1} x(s) d s
$$

where $n=[\alpha]+1,[\alpha]$ denotes the integer part of number $\alpha$, provided that the right-hand side is pointwise defined on $(0,+\infty)$.

Proposition 2.3 (see [21, 22]). (1) If $x \in L^{1}(0,1), v>\sigma>0$, then

$$
I^{v} I^{\sigma} x(t)=I^{v+\sigma} x(t), \quad \Phi_{\mathrm{t}}^{\sigma} I^{v} x(t)=I^{v-\sigma} x(t), \quad \Phi_{\mathrm{t}}^{\sigma} I^{\sigma} x(t)=x(t)
$$

(2) If $\alpha>0, \sigma>0$, then

$$
\Phi_{\mathfrak{t}}^{\alpha} t^{\sigma-1}=\frac{\Gamma(\sigma)}{\Gamma(\sigma-\alpha)} t^{\sigma-\alpha-1}
$$

Proposition 2.4 (see $[21,22]$ ). Let $\alpha>0$, and $f(x)$ is integrable, then

$$
I^{\alpha} \boldsymbol{\oplus}_{\mathfrak{t}}^{\alpha} f(x)=f(x)+c_{1} x^{\alpha-1}+c_{2} x^{\alpha-2}+\cdots+c_{n} x^{\alpha-n},
$$

where $c_{i} \in \mathbb{R}(i=1,2, \ldots, n), n$ is the smallest integer greater than or equal to $\alpha$.

Our discussion is based on the assumption $-1<\alpha-n \leq 0$ in this paper.

Let $x(t)=I^{n-2} y(t), y(t) \in C[0,1]$, by standard discuss, we easily reduce order the BVP (1.4) to the following equivalent BVP

$$
\begin{gathered}
-\Phi_{\mathfrak{t}}{ }^{\alpha-n+2} y(t)=f\left(t, I^{n-2} y(t), I^{n-3} y(t), \ldots, I^{1} y(t), y(t)\right), \\
y(0)=0, \quad y(1)=\int_{0}^{1} y(s) d A(s) .
\end{gathered}
$$


In fact, let $x(t)=I^{n-2} y(t), y(t) \in C[0,1]$, then

$$
\begin{gathered}
\Phi_{\mathfrak{t}}^{\alpha} x(t)=\frac{d^{n}}{d t^{n}} I^{n-\alpha} x(t)=\frac{d^{n}}{d t^{n}} I^{n-\alpha} I^{n-2} y(t) \\
=\frac{d^{n}}{d t^{n}} I^{2 n-\alpha-2} y(t)=\Phi_{\mathfrak{t}}{ }^{\alpha-n+2} y(t), \\
x^{\prime}(t)=\Phi_{\mathfrak{t}}^{1} I^{n-2} y(t)=I^{n-3} y(t), \\
x^{\prime \prime}(t)=\Phi_{\mathfrak{t}}^{2} I^{n-2} y(t)=I^{n-4} y(t), \\
\vdots \\
x^{(n-3)}(t)=\Phi_{\mathfrak{t}}^{n-3} I^{n-2} y(t)=I^{1} y(t) \\
x^{(n-2)}(t)=\Phi_{\mathfrak{t}}{ }^{n-2} I^{n-2} y(t)=y(t) .
\end{gathered}
$$

By (2.7), we have $x^{(n-2)}(0)=y(0)=0$ and

$$
y(1)=\int_{0}^{1} y(s) d A(s)
$$

Moreover $-\Phi_{\mathbf{t}}^{\alpha-n+2} y(t)=f\left(t, I^{n-2} y(t), I^{n-3} y(t), \ldots, I^{1} y(t), y(t)\right)$. Thus, (1.4) is transformed into (2.6).

On the other hand, if $y \in C([0,1],[0,+\infty))$ is a solution for problem (2.6). Then, from Proposition 2.3 and (2.7), one has

$$
\begin{aligned}
-\Phi_{\mathfrak{t}}{ }^{\alpha} x(t) & =-\Phi_{\mathfrak{t}}{ }^{\alpha-n+2} y(t)=f\left(t, I^{n-2} y(t), I^{n-3} y(t), \ldots, I^{1} y(t), y(t)\right) \\
& =f\left(t, x(t), x^{\prime}(t), x^{\prime \prime}(t), \ldots, x^{(n-2)}(t)\right), \quad 0<t<1 .
\end{aligned}
$$

Notice for any $\alpha>0$,

$$
I^{\alpha} y(t)=\frac{1}{\Gamma(\alpha)} \int_{0}^{t}(t-s)^{\alpha-1} y(s) d s
$$

which implies that $I^{\alpha} y(0)=0$. So $x(0)=0$, and from (2.7), for $i=1,2, \ldots, n-2$, we have $x^{(i)}(0)=0$, and

$$
x^{(n-2)}(1)=\int_{0}^{1} x^{(n-2)}(s) d A(s) .
$$

Consequently, the BVP (2.6) is transformed into the BVP (1.4).

Applying Propositions 2.3 and 2.4 , by standard discuss, we have the following Lemma. 
Lemma 2.5. Given $y \in L^{1}(0,1)$, then the problem

$$
\begin{gathered}
\Phi_{\mathrm{t}}^{\alpha-n+2} y(t)+h(t)=0, \quad 0<t<1, \\
y(0)=0, \quad y(1)=0
\end{gathered}
$$

has the unique solution

$$
y(t)=\int_{0}^{1} G(t, s) h(s) d s,
$$

where $G(t, s)$ is the Green function of the BVP (2.12) and is given by the following:

$$
G(t, s)= \begin{cases}\frac{t^{\alpha-n+1}(1-s)^{\alpha-n+1}-(t-s)^{\alpha-n+1}}{\Gamma(\alpha-n+2)}, & 0 \leq s \leq t \leq 1 \\ \frac{t^{\alpha-n+1}(1-s)^{\alpha-n+1}}{\Gamma(\alpha-n+2)}, & 0 \leq t \leq s \leq 1\end{cases}
$$

By Proposition 2.4, the unique solution of the problem

$$
\begin{gathered}
\Phi_{\mathrm{t}}^{\alpha-n+2} y(t)=0, \quad 0<t<1, \\
y(0)=0, \quad y(1)=1,
\end{gathered}
$$

is $t^{\alpha-n+1}$. Let

$$
\mathcal{C}=\int_{0}^{1} t^{\alpha-n+1} d A(t)
$$

and define

$$
\mathcal{G}_{A}(s)=\int_{0}^{1} G(t, s) d A(t) .
$$

Then the Green function for the nonlocal BVP (2.6) is (the detail see [23] or [16])

$$
K(t, s)=\frac{t^{\alpha-n+1}}{1-\mathcal{C}} \mathcal{G}_{A}(s)+G(t, s)
$$

Throughout paper, we always assume the following holds. 
(H0) $A$ is a function of bounded variation such that $\mathcal{G}_{A}(s) \geq 0$ for $s \in[0,1]$ and $0 \leq \mathcal{C}<$ 1 , where $\mathcal{C}$ is defined by (2.16).

Lemma 2.6. Suppose (H0) holds, then the Green function defined by (2.18) satisfies

(1) $K(t, s)>0$, for all $t, s \in(0,1)$.

(2)

$$
\frac{t^{\alpha-n+1}}{1-\mathcal{C}} \mathcal{G}_{A}(s) \leq K(t, s) \leq \mathscr{H}(s) t^{\alpha-n+1}
$$

where

$$
\mathscr{H}(s)=\frac{(1-s)^{\alpha-n+1}}{\Gamma(\alpha-n+2)}+\frac{\mathcal{G}_{A}(s)}{1-\mathcal{C}}
$$

Proof. (1) is obvious. For (2), by (2.14) and (2.18), we have

$$
\begin{aligned}
H(t, s) & =\frac{t^{\alpha-n+1}}{1-\mathcal{C}} \mathcal{G}_{A}(s)+G(t, s) \leq \frac{t^{\alpha-n+1}(1-s)^{\alpha-n+1}}{\Gamma(\alpha-n+2)}+\frac{t^{\alpha-n+1}}{1-\mathcal{C}} \mathcal{G}_{A}(s) \\
& =\mathscr{H}(s) t^{\alpha-n+1}, \\
H(t, s) & =\frac{t^{\alpha-n+1}}{1-\mathcal{C}} \mathcal{G}_{A}(s)+G(t, s) \geq \frac{t^{\alpha-n+1}}{1-\mathcal{C}} \mathcal{G}_{A}(s) .
\end{aligned}
$$

Definition 2.7. A continuous function $\psi(t)$ is called a lower solution of the BVP (2.6), if it satisfies

$$
\begin{gathered}
-\Phi_{\mathrm{t}}{ }^{\alpha-n+2} \psi(t)(t) \leq f\left(t, I^{n-2} \psi(t), I^{n-3} \psi(t), \ldots, I^{1} \psi(t), \psi(t)\right), \\
\psi(0) \geq 0, \quad \psi(1) \geq \int_{0}^{1} \psi(s) d A(s) .
\end{gathered}
$$

Definition 2.8. A continuous function $\phi(t)$ is called a upper solution of the BVP (2.6), if it satisfies

$$
\begin{gathered}
-\Phi_{\mathfrak{t}}^{\alpha-n+2} \phi(t)(t) \geq f\left(t, I^{n-2} \phi(t), I^{n-3} \phi(t), \ldots, I^{1} \phi(t), \phi(t)\right), \\
\phi(0) \leq 0, \quad \phi(1) \leq \int_{0}^{1} \phi(s) d A(s) .
\end{gathered}
$$

It follows from Lemma 2.5, we have the following maximum principle. 
Lemma 2.9 (maximum principle). If $y \in C([0,1], \mathbb{R})$ satisfies

$$
y(0)=0, \quad y(1)=\int_{0}^{1} y(s) d A(s),
$$

and $\Phi_{\mathbf{t}}{ }^{\alpha-n+2} y(t) \leq 0$ for any $t \in(0,1)$. Then

$$
y(t) \geq 0, \quad t \in[0,1]
$$

\section{Main Results}

Let $E=C[0,1]$, and

$$
\begin{gathered}
P=\left\{y \in E: \text { there exist positive numbers } 0<l_{y}<1, L_{y}>1\right. \\
\text { such that } \left.l_{y} t^{\alpha-n+1} \leq y(t) \leq L_{y} t^{\alpha-n+1}, t \in[0,1]\right\}
\end{gathered}
$$

Clearly, $t^{\alpha-n+1} \in P$, so $P$ is nonempty. For any $y \in P$, define an operator $T$ by the following:

$$
(T y)(t)=\int_{0}^{1} K(t, s) f\left(s, I^{n-2} y(s), I^{n-3} y(s), \ldots, I^{1} y(s), y(s)\right) d s
$$

Let

$$
\kappa_{i}(t)=\frac{\Gamma(\alpha-n+2)}{\Gamma(\alpha-i)} t^{\alpha-i-1}, \quad i=0,1,2, \ldots, n-2,
$$

then

$$
\kappa_{0}(t)=I^{n-2} s^{\alpha-n+1}, \kappa_{1}(t)=I^{n-3} s^{\alpha-n+1}, \ldots, \kappa_{n-3}(t)=I^{1} s^{\alpha-n+1}, \kappa_{n-2}(t)=t^{\alpha-n+1} .
$$

The conditions imposed on $f$ are the following.

(H1) $f \in C\left((0,1) \times(0, \infty)^{n-1},[0,+\infty)\right)$, and $f\left(t, x_{0}, x_{1}, x_{2}, \ldots, x_{n-2}\right)$ is decreasing in $x_{i}>0$ for $i=0,1,2, \ldots, n-2$;

(H2) for any $\lambda_{i}>0, f\left(t, \lambda_{0} t^{n-2}, \lambda_{1} t^{n-3}, \ldots, \lambda_{n-3} t, \lambda_{n-2}\right) \neq \equiv, t \in(0,1)$, and

$$
0<\int_{0}^{1} \mathscr{H}(s) f\left(s, \lambda_{0} \kappa_{0}(s), \lambda_{1} \kappa_{1}(s), \lambda_{2} \kappa_{2}(s), \ldots, \lambda_{n-2} \kappa_{n-2}(s)\right) d s<+\infty .
$$


Lemma 3.1. Suppose (H0)-(H2) hold, then $T$ is well defined and $T(P) \subset P$.

Proof. For any $y \in P$, by the definition of $P$, there exists two positive numbers $0<l_{y}<1, L_{y}>$ 1 such that $l_{y} t^{\alpha-n+1} \leq y(t) \leq L_{y} t^{\alpha-n+1}$ for any $t \in[0,1]$. It follows from (2.19) and (H1)-(H2) that

$$
\begin{aligned}
(T y)(t) & =\int_{0}^{1} K(t, s) f\left(s, I^{n-2} y(s), I^{n-3} y(s), \ldots, I^{1} y(s), y(s)\right) d s \\
& \leq \int_{0}^{1} \mathscr{H}(s) f\left(s, I^{n-2} l_{y} s^{\alpha-n+1}, \ldots, I^{1} l_{y} s^{\alpha-n+1}, l_{y} s^{\alpha-n+1}\right) d s \\
& =\int_{0}^{1} \mathscr{H}(s) f\left(s, l_{y} \kappa_{0}(s), l_{y} \kappa_{1}(s), \ldots, l_{y} \kappa_{n-2}(s)\right) d s<+\infty
\end{aligned}
$$

Now take $C=\max _{t \in[0,1]} y(t)$, then for any $s \in(0,1)$,

$$
\mathcal{G}_{A}(s) f\left(s, \frac{C}{(n-2) !} s^{n-2}, \frac{C}{(n-3) !} s^{n-3}, \ldots, \frac{C}{1 !} s, C\right) \not \equiv 0,
$$

by (H2). Thus by the continuity of $f\left(t, x_{0}, x_{1}, \ldots, x_{n-2}\right)$, we have

$$
\int_{0}^{1} \mathcal{G}_{A}(s) f\left(s, \frac{C}{(n-2) !} s^{n-2}, \frac{C}{(n-3) !} s^{n-3}, \ldots, \frac{C}{1 !} s, C\right) d s>0 .
$$

This yields

$$
\begin{aligned}
& \int_{0}^{1} \mathcal{G}_{A}(s) f\left(s, I^{n-2} C, I^{n-3} C, \ldots, I^{1} C, C\right) d s \\
& \quad=\int_{0}^{1} \mathcal{G}_{A}(s) f\left(s, \frac{C}{(n-2) !} s^{n-2}, \frac{C}{(n-3) !} s^{n-3}, \ldots, \frac{C}{1 !} s, C\right) d s>0 .
\end{aligned}
$$

By (2.19) and (3.6)-(3.9), we have

$$
\begin{aligned}
(T y)(t) & =\int_{0}^{1} K(t, s) f\left(s, I^{n-2} y(s), I^{n-3} y(s), \ldots, I^{1} y(s), y(s)\right) d s \\
& \geq \frac{t^{\alpha-n+1}}{1-C} \int_{0}^{1} \mathcal{G}_{A}(s) f\left(s, I^{n-2} C, I^{n-3} C, \ldots, I^{1} C, C\right) d s \geq l_{y}^{\prime} t^{\alpha-n+1}
\end{aligned}
$$

where

$$
l_{y}^{\prime}=\min \left\{1, \frac{1}{1-\mathcal{C}} \int_{0}^{1} \mathcal{G}_{A}(s) f\left(s, I^{n-2} C, I^{n-3} C, \ldots, I^{1} C, C\right) d s\right\}
$$


On the other hand, it follows from (2.19) that

$$
\begin{aligned}
(T y)(t) & =\int_{0}^{1} K(t, s) f\left(s, I^{n-2} y(s), I^{n-3} y(s), \ldots, I^{1} y(s), y(s)\right) d s \\
& \leq t^{\alpha-n+1} \int_{0}^{1} \mathscr{H}(s) f\left(s, l_{y} \kappa_{0}(s), l_{y} \kappa_{1}(s), \ldots, l_{y} \kappa_{n-2}(s)\right) d s \\
& \leq L_{y}^{\prime} t^{\alpha-n+1}
\end{aligned}
$$

where

$$
L_{y}^{\prime}=\max \left\{1, \int_{0}^{1} \mathscr{H}(s) f\left(s, l_{y} \kappa_{0}(s), l_{y} \kappa_{1}(s), \ldots, l_{y} \kappa_{n-2}(s)\right) d s\right\} .
$$

It follows from (3.6)-(3.12) that $T$ is well defined and $T(P) \subset P$.

Take

$$
\begin{gathered}
L=\max \left\{1, \int_{0}^{1} \mathscr{H}(s) f\left(s, \kappa_{0}(s), \kappa_{1}(s), \ldots, \kappa_{n-2}(s)\right) d s\right\} \\
l=\min \left\{1, \frac{1}{1-\mathcal{C}} \int_{0}^{1} \mathcal{G}_{A}(s) f\left(s, \kappa_{0}(s), \kappa_{1}(s), \ldots, \kappa_{n-2}(s)\right) d s\right\} .
\end{gathered}
$$

By (H2) and Weierstrass distinguishing method, we know

$$
a(t)=\int_{0}^{1} K(t, s) f\left(s, \kappa_{0}(s), \kappa_{1}(s), \ldots, \kappa_{n-2}(s)\right) d s
$$

is a continuous function on $[0,1]$, that is, $a(t) \in E$.

Theorem 3.2 (existence). Suppose (H0)-(H2) hold, and if

$$
\int_{0}^{1} \mathcal{G}_{A}(s) f\left(s, \frac{L}{l} \kappa_{0}(s), \frac{L}{l} \kappa_{1}(s), \ldots, \frac{L}{l} \kappa_{n-2}(s), \frac{L}{l} \kappa_{n-1}(s)\right) d s \geq 1-\mathcal{C} .
$$

Then the BVP (1.4) has at least a positive solution $w(t)$.

Proof. By (2.18) and (3.2), we have

$$
\begin{gathered}
-\Phi_{\mathfrak{t}}{ }^{\alpha-n+2}(T y)(t)=f\left(t, I^{n-2} y(t), I^{n-3} y(t), \ldots, I^{1} y(t), y(t)\right), \\
(T y)(0)=0, \quad(T y)(1)=\int_{0}^{1}(T y)(s) d A(s) .
\end{gathered}
$$


Abstract and Applied Analysis

It follows from (2.19) that

$$
l t^{\alpha-n+1} \leq a(t) \leq L t^{\alpha-n+1},
$$

which implies that $a(t) \in P$. Let

$$
b(t)=\frac{a(t)}{l},
$$

then we also have

$$
t^{\alpha-n+1} \leq b(t) \leq \frac{L}{l} t^{\alpha-n+1}
$$

Thus according to the fact that the operator $T$ is nonincreasing relative to $y$, Lemma 3.1 and (3.20), we have

$$
T b(t) \leq T t^{\alpha-n+1}=a(t) \leq \frac{a(t)}{l}=b(t) .
$$

and $T b(t) \in P$. By (2.19), (3.12), and (3.20), one has

$$
\begin{aligned}
T b(t) & \geq \frac{1}{1-\mathcal{C}} t^{\alpha-n+1} \int_{0}^{1} \mathcal{G}_{A}(s) f\left(s, I^{n-2} b(s), I^{n-3} b(s), \ldots, I^{1} b(s), b(s)\right) d s \\
& \geq \frac{1}{1-\mathcal{C}} t^{\alpha-n+1} \int_{0}^{1} \mathcal{G}_{A}(s) f\left(s, \frac{L}{l} \kappa_{0}(s), \frac{L}{l} \kappa_{1}(s), \ldots, \frac{L}{l} \kappa_{n-2}(s)\right) d s \\
& \geq t^{\alpha-n+1} .
\end{aligned}
$$

Thus, by (3.20), (3.21) and the operator $T$ is nonincreasing relative to $y$, we have

$$
\begin{aligned}
& \Phi_{\mathrm{t}}^{\alpha-n+2} b(t)+f\left(t, I^{n-2} b(t), I^{n-3} b(t), \ldots, I^{1} b(t), b(t)\right) \\
& =-\frac{1}{l} f\left(s, I^{n-2} t^{\alpha-n+1}, \ldots, I^{1} t^{\alpha-n+1}, t^{\alpha-n+1}\right)+f\left(t, I^{n-2} b(t), \ldots, I^{1} b(t), b(t)\right) \\
& \leq-\frac{1}{l} f\left(s, I^{n-2} t^{\alpha-n+1}, \ldots, I^{1} t^{\alpha-n+1}, t^{\alpha-n+1}\right)+f\left(s, I^{n-2} t^{\alpha-n+1}, \ldots, I^{1} t^{\alpha-n+1}, t^{\alpha-n+1}\right) \\
& \leq 0 \text {, } \\
& \Phi_{\mathfrak{t}}^{\alpha-n+2} \mathrm{~Tb}(t)+f\left(t, I^{n-2} \mathrm{~Tb}(t), I^{n-3} \mathrm{~Tb}(t), \ldots, I^{1} \mathrm{~Tb}(t), T b(t)\right) \\
& =-f\left(t, I^{n-2} b(t), I^{n-3} b(t), \ldots, I^{1} b(t), b(t)\right) \\
& +f\left(t, I^{n-2} T b(t), I^{n-3} T b(t), \ldots, I^{1} T b(t), T b(t)\right) \\
& \geq 0, \quad t \in[0,1] \text {. }
\end{aligned}
$$


However (3.17) implies that $b(t), T b(t)$ satisfy boundary conditions of BVP (2.6). Let

$$
\psi(t)=T b(t), \quad \phi(t)=\frac{1}{l} T t^{\alpha-n+1}=\frac{1}{l} a(t)=b(t),
$$

then (3.21), (3.23) imply that $\psi(t), \phi(t)$ are lower and upper solution of BVP (2.6), respectively, and $\psi(t), \phi(t) \in P$.

Define the function $F$ and the operator $A$ in $E$ by the following:

$$
\begin{gathered}
F(t, y)= \begin{cases}f\left(t, I^{n-2} \psi(t), I^{n-3} \psi(t), \ldots, I^{1} \psi(t), \psi(t)\right), & y<\psi(t), \\
f\left(t, I^{n-2} y(t), I^{n-3} y(t), \ldots, I^{1} y(t), y(t)\right), & \psi(t) \leq y \leq \phi(t), \\
f\left(t, I^{n-2} \phi(t), I^{n-3} \phi(t), \ldots, I^{1} \phi(t), \phi(t)\right), & y>\phi(t),\end{cases} \\
(B y)(t)=\int_{0}^{1} K(t, s) F(s, y(s)) d s, \quad \forall y \in E .
\end{gathered}
$$

Clearly, $F:[0,1] \times[0,+\infty) \rightarrow[0,+\infty)$ is continuous by (3.25). Consider the following boundary value problem

$$
\begin{aligned}
& -\Phi_{\mathbf{t}}^{\alpha-n+2} y(t)=F(t, y), \quad 0<t<1, \\
& y(0)=0, \quad y(1)=\int_{0}^{1} y(s) d A(s) .
\end{aligned}
$$

Obviously, a fixed point of the operator $B$ is a solution of the BVP (3.27).

For all $y \in E$, it follows from Lemma 2.6 and (3.22) that

$$
\begin{aligned}
(B y)(t) & \leq \int_{0}^{1} \mathscr{H}(s) F(s, y(s)) d s \leq \int_{0}^{1} \mathscr{H}(s) f\left(s, I^{n-2} \psi(s), I^{n-3} \psi(s), \ldots, I^{1} \psi(s), \psi(s)\right) d s \\
& \leq \int_{0}^{1} \mathscr{H}(s) f\left(s, I^{n-2} s^{\alpha-n+1}, \ldots, I^{1} s^{\alpha-n+1}, s^{\alpha-n+1}\right) d s \\
& =\int_{0}^{1} \mathscr{H}(s) f\left(s, \kappa_{0}(s), \kappa_{1}(s), \ldots, \kappa_{n-2}(s)\right) d s<+\infty .
\end{aligned}
$$

So $B$ is bounded. It is easy to see $B: E \rightarrow E$ is continuous from the continuity of $F$ and $K$.

Let $\Omega \subset E$ be bounded, that is, there exists a positive constant $N>0$ such that $\|y\| \leq N$, for all $y \in \Omega$. Let $L=\max _{0 \leq t \leq 1,0 \leq y \leq N}|F(t, y)|+1$, and since $K(t, s)$ is uniformly continuous on $[0,1] \times[0,1]$, then for any $\varepsilon>0$ and $s \in[0,1]$, there exists $\delta>0$ such that

$$
\left|K\left(t_{1}, s\right)-K\left(t_{2}, s\right)\right|<\frac{\varepsilon}{L}
$$


for $\left|t_{1}-t_{2}\right|<\delta$. Then

$$
\left|B y\left(t_{1}\right)-B y\left(t_{2}\right)\right| \leq \int_{0}^{1}\left|K\left(t_{1}, s\right)-K\left(t_{2}, s\right)\right||F(s, y(s))| d s<\varepsilon
$$

This implies that $B(\Omega)$ is equicontinuous.

By the means of the Arzela-Ascoli theorem, we have $B: E \rightarrow E$ is completely continuous. Thus, by using Schauder's fixed-point theorem, $B$ has at least a fixed-point $w$ such that $w=B w$.

Now we prove

$$
\psi(t) \leq w(t) \leq \phi(t), \quad t \in[0,1] .
$$

Let $z(t)=\phi(t)-w(t), t \in[0,1]$. By $\phi(t)$ is upper solution of BVP (2.6) and $w$ is fixed point of $B$, we know

$$
w(0)=0, \quad w(1)=\int_{0}^{1} w(s) d A(s)
$$

From the definition of $F$ and (3.22), we obtain

$$
\begin{aligned}
f(t, & \left.I^{n-2} \phi(t), I^{n-3} \phi(t), \ldots, I^{1} \phi(t), \phi(t)\right) \leq F(t, y(t)) \\
& \leq f\left(t, I^{n-2} \psi(t), I^{n-3} \psi(t), \ldots, I^{1} \psi(t), \psi(t)\right) \\
& \leq f\left(t, I^{n-2} t^{\alpha-n+1}, \ldots, I^{1} t^{\alpha-n+1}, t^{\alpha-n+1}\right), \quad t \in[0,1] .
\end{aligned}
$$

Thus (3.16) and (3.33) imply

$$
\begin{aligned}
\Phi_{\mathrm{t}}^{\alpha-n+2} z(t)=\Phi_{\mathrm{t}}^{\alpha-n+2} \phi(t)-\Phi_{\mathrm{t}}^{\alpha-n+2} w(t) \\
=-\frac{1}{l} f\left(t, I^{n-2} t^{\alpha-n+1}, \ldots, I^{1} t^{\alpha-n+1}, t^{\alpha-n+1}\right)+F(t, w(t)) \\
\leq-\frac{1}{l} f\left(t, I^{n-2} t^{\alpha-n+1}, \ldots, I^{1} t^{\alpha-n+1}, t^{\alpha-n+1}\right) \\
\quad+f\left(t, I^{n-2} t^{\alpha-n+1}, \ldots, I^{1} t^{\alpha-n+1}, t^{\alpha-n+1}\right) \leq 0, \quad \forall t \in[0,1] .
\end{aligned}
$$

By (3.32), (3.34), and Lemma 2.9, we know $z(t) \geq 0$ which implies $w(t) \leq \phi(t)$ on $[0,1]$. By the same way, it is easy to prove $w(t) \geq \psi(t)$ on $[0,1]$. So we obtain

$$
\psi(t) \leq w(t) \leq \phi(t), \quad t \in[0,1] .
$$


Consequently, $F(t, w(t))=f\left(t, I^{n-2} w(t), I^{n-3} w(t), \ldots, I^{1} w(t), w(t)\right), t \in[0,1]$. Then $w(t)$ is a positive solution of the BVP (2.6), which implies that $x(t)=I^{n-2} w(t)$ is a positive solution of the BVP (1.4).

Theorem 3.3 (asymptotic behavior). Suppose the conditions of Theorem 3.2 are satisfied. Then there exist two constants $\boldsymbol{B}_{1}, \boldsymbol{B}_{2}$ such that the positive solution $x(t)$ of the BVP (1.4) satisfies

$$
\begin{gathered}
\mathbb{B}_{1} t^{\alpha-1} \leq x(t) \leq \boldsymbol{B}_{2} t^{\alpha-1}, \\
\lim _{t \rightarrow 0+} \frac{x(t)}{t^{\alpha-1}}=0
\end{gathered}
$$

where

$$
B_{1}=\frac{\Gamma(\alpha-n+2)}{\Gamma(\alpha)}, \quad B_{2}=L B_{1} .
$$

Proof. By (3.22) and (3.35), we know

$$
w(t) \geq \psi(t) \geq t^{\alpha-n+1}
$$

On the other hand, it follows from (3.38) and (2.19) that

$$
\begin{aligned}
w(t) & =\int_{0}^{1} K(t, s) f\left(s, I^{n-2} w(s), I^{n-3} w(s), \ldots, I^{1} w(s), w(s)\right) d s \\
& \leq t^{\alpha-n+1} \int_{0}^{1} \mathscr{H}(s) f\left(s, I^{n-2} s^{\alpha-n+1}, \ldots, I^{1} s^{\alpha-n+1}, s^{\alpha-n+1}\right) d s \\
& =t^{\alpha-n+1} \int_{0}^{1} \mathscr{H}(s) f\left(s, \kappa_{0}(s), \kappa_{1}(s), \ldots, \kappa_{n-2}(s), \kappa_{n-1}(s)\right) d s \\
& \leq L t^{\alpha-n+1} .
\end{aligned}
$$

Then

$$
t^{\alpha-n+1} \leq w(t) \leq L t^{\alpha-n+1}
$$

Since

$$
I^{n-2} t^{\alpha-n+1}=\frac{1}{\Gamma(n-2)} \int_{0}^{t}(t-s)^{n-3} s^{\alpha-n+1} d s=\frac{\Gamma(\alpha-n+2)}{\Gamma(\alpha)} t^{\alpha-1},
$$

so it follows from (3.40) that

$$
\frac{\Gamma(\alpha-n+2)}{\Gamma(\alpha)} t^{\alpha-1} \leq I^{n-2} w(t)=x(t) \leq L \frac{\Gamma(\alpha-n+2)}{\Gamma(\alpha)} t^{\alpha-1},
$$


That is,

$$
\mathfrak{B}_{1} t^{\alpha-1} \leq x(t) \leq \mathbb{B}_{2} t^{\alpha-1}
$$

In the end, from $n-1<\alpha \leq n$ and L'Hospital Rule, we have

$$
\begin{aligned}
\operatorname{Lim}_{t \rightarrow 0+} \frac{x(t)}{t^{\alpha-1}} & =\lim _{t \rightarrow 0+} \frac{x^{(n-2)}(t)}{(\alpha-1)(\alpha-2) \cdots(\alpha-n+2) t^{\alpha-n+1}} \\
& =\lim _{t \rightarrow 0+} \frac{x^{(n-1)}(t)}{(\alpha-1)(\alpha-2) \cdots(\alpha-n+1) t^{\alpha-n}}=0 .
\end{aligned}
$$

Theorem 3.4 (uniqueness). Suppose the conditions of Theorem 3.2 are satisfied and $\alpha=n$. Then the positive solution $x(t)$ of the BVP (1.4) is unique.

Proof. We only need the BVP (2.6) has unique solution. Suppose that $w_{1}, w_{2}$ are $C[0,1]$ positive solutions to the BVP (2.6). We may assume, without loss of generality, that there exists $t^{*} \in(0,1)$ such that $w_{2}\left(t^{*}\right)-w_{1}\left(t^{*}\right)=\max \left\{w_{2}(t)-w_{1}(t)\right\}>0$. Let

$$
\begin{aligned}
a & =\inf \left\{t_{1} \mid 0 \leq t_{1}<t^{*}, w_{2}(t) \geq w_{1}(t), t \in\left(t_{1}, t^{*}\right]\right\} ; \\
b & =\inf \left\{t_{2} \mid t^{*}<t_{2} \leq 1, w_{2}(t) \geq w_{1}(t), t \in\left(t^{*}, t_{2}\right]\right\} ; \\
z(t) & =w_{2}(t)-w_{1}(t), \quad t \in[0,1] .
\end{aligned}
$$

Evidently,

$$
\begin{aligned}
t^{*} \in(a, b), \quad w_{2}(t) \geq w_{1}(t), \quad f & \left(t, I^{n-2} w_{2}(t), I^{n-3} w_{2}(t), \ldots, I^{1} w_{2}(t), w_{2}(t)\right) \\
& \leq f\left(t, I^{n-2} w_{1}(t), I^{n-3} w_{1}(t), \ldots, I^{1} w_{1}(t), w_{2}(t)\right), \quad t \in[a, b] .
\end{aligned}
$$

Thus

$$
\Phi_{\mathbf{t}}^{\alpha-n+2} z(t)=z^{\prime \prime}(t) \geq 0, \quad t \in[a, b]
$$

By the boundary conditions of the BVP (2.6), it is easy to check that there exist the following two possible cases:

$$
\begin{array}{ll}
\text { (1) } z(a)=0, & z(b)=0 \\
\text { (2) } z(a)=0, & z(b)>0
\end{array}
$$

Case 1. From $z^{\prime \prime}(t) \geq 0$ and $z(a)=0, z(b)=0$, we get that $z(t) \leq 0, t \in[a, b]$, which implies a contradiction with $w_{2}\left(t^{*}\right)>w_{1}\left(t^{*}\right)$. 
Case 2. In this case we have $b=1$ and $z^{\prime}\left(t^{*}\right)=0$. Since $z^{\prime}(t)$ is increasing on $[a, b]$, we also have $z^{\prime}(t)$ is increasing on $t \in\left[a, t^{*}\right]$, that is, $z^{\prime}(t) \leq z^{\prime}\left(t^{*}\right)=0$ for any $t \in\left[a, t^{*}\right]$. Thus $z(t)$ is nonincreasing on $t \in\left[a, t^{*}\right]$, which implies that $z\left(t^{*}\right) \leq z(a)=0$, this is a contradiction with $w_{2}\left(t^{*}\right)>w_{1}\left(t^{*}\right)$.

Therefore the BVP (2.6) has unique solution, and the BVP (1.4) also has unique solution.

Remark 3.5. We only get the uniqueness of positive solution for the BVP (1.4) as $\alpha$ is integer, but it remains unknown as to whether an analogous unique result holds for the case $n-1<$ $\alpha<n$. We believe the result should also hold and try to study this problem, but it is a pity that we failed to find the effective method to solve this problem.

Remark 3.6. The BVP (1.4) allows the nonlinearity $f\left(t, x_{0}, x_{2}, \ldots, x_{n-2}\right)$ has singularity at $t=$ 0,1 and $x_{i}=0, i=0,1, \ldots, n-2$. If $f\left(t, x_{0}, x_{2}, \ldots, x_{n-2}\right)$ is continuous at $x_{i}=0, i=0,1, \ldots, n-2$, that is

$f \in C\left((0,1) \times[0, \infty)^{n-1},[0,+\infty)\right)$, and $f\left(t, x_{0}, x_{1}, x_{2}, \ldots, x_{n-2}\right)$ is decreasing in $x_{i}>0$ for $i=0,1,2, \ldots, n-2$;

$$
0<\int_{0}^{1} \mathscr{H}(s) f(s, 0,0, \ldots, 0) d s<+\infty
$$

then the BVP (1.4) has at least a positive solution $x(t)$, and there exists a constant $B_{3}>0$ such that

$$
0 \leq x(t) \leq B_{3} t^{\alpha-1}
$$

If $\alpha=n$, the positive solution of the BVP (1.4) also is unique.

Proof. In fact, in Theorem 3.2 the set $P$ is replaced by the following:

$$
P_{1}=\{x \in E: x(t) \geq 0, t \in[0,1]\}
$$

and (3.21) can be replaced by the following:

$$
0 \leq \phi(t)=T(0), \quad 0 \leq \psi(t)=(T \phi)(t) \leq T(0)=\phi(t) .
$$

Clearly $\phi(t), \psi(\mathrm{t}) \in P_{1}$, and

$$
\begin{aligned}
& \boldsymbol{\Phi}_{\mathrm{t}}^{\alpha-n+2} \phi(t)+f\left(t, I^{n-2} \phi(t), I^{n-3} \phi(t), \ldots, I^{1} \phi(t), \phi(t)\right) \\
& \quad=-f(t, 0,0, \ldots, 0)+f\left(t, I^{n-2} T(0), I^{n-3} T(0), \ldots, I^{1} T(0), T(0)\right) \leq 0,
\end{aligned}
$$




$$
\begin{aligned}
& \bigoplus_{\mathfrak{t}}^{\alpha-n+2} \psi(t)+f\left(t, I^{n-2} \psi(t), I^{n-3} \psi(t), \ldots, I^{1} \psi(t), \psi(t)\right) \\
& \quad=-f\left(t, I^{n-2} \phi(t), I^{n-3} \phi(t), \ldots, I^{1} \phi(t), \phi(t)\right)+f\left(t, I^{n-2} \psi(t), I^{n-3} \psi(t), \ldots, I^{1} \psi(t), \psi(t)\right) \\
& \quad \geq 0, \quad t \in[0,1] .
\end{aligned}
$$

Thus the rest of proof is similar to those of Theorem 3.2.

On the other hand, by Lemma 2.6 and (G2), the positive solution of the BVP (2.6) satisfies

$$
\begin{aligned}
0 & \leq w(t) \leq \phi(t)=T 0=\int_{0}^{1} K(t, s) f(s, 0,0, \ldots, 0) d s \\
& \leq \int_{0}^{1} \mathscr{H}(s) f(s, 0,0, \ldots, 0) d s t^{\alpha-n+1},
\end{aligned}
$$

and then

$$
0 \leq I^{n-2} w(t)=x(t) \leq \boldsymbol{B}_{3} t^{\alpha-1}
$$

where

$$
\mathbb{B}_{3}=\frac{\Gamma(\alpha-n+2)}{\Gamma(\alpha)} \int_{0}^{1} \mathscr{H}(s) f(s, 0,0, \ldots, 0) d s
$$

Moreover if $\alpha=n$, the positive solution of the BVP (1.4) also is unique.

Example 3.7. Consider the existence of positive solutions for the nonlinear fractional differential equation

$$
\begin{gathered}
-\Phi_{\mathfrak{t}}^{7 / 2} x(t)=10 t^{-1 / 4}\left[\left(x^{\prime}\right)^{-1 / 3}+x^{-1 / 8}\right], \quad 0<t<1, \\
x(0)=x^{\prime}(0)=0, \quad x^{\prime}(1)=\int_{0}^{1} x^{\prime}(s) d A(s),
\end{gathered}
$$

where

$$
A(t)= \begin{cases}0, & t \in\left[0, \frac{1}{2}\right) \\ \frac{3}{2}, & t \in\left[\frac{1}{2}, \frac{3}{4}\right) \\ 1, & t \in\left[\frac{3}{4}, 1\right] .\end{cases}
$$


Then the BVP (3.58) is equivalent to the following 4-point BVP with coefficients of both signs

$$
\begin{aligned}
& -\Phi_{\mathfrak{t}}^{7 / 2} x(t)=10 t^{-1 / 4}\left[\left(x^{\prime}\right)^{-1 / 3}+x^{-1 / 8}\right], \quad 0<t<1, \\
& x(0)=x^{\prime}(0)=0, \quad x^{\prime}(1)=\frac{3}{2} x^{\prime}\left(\frac{1}{2}\right)-\frac{1}{2} x^{\prime}\left(\frac{3}{4}\right),
\end{aligned}
$$

Conclusion: The BVP (3.59) has at least a positive solution $x(t)$ such that

$$
\frac{2}{5} t^{5 / 2} \leq x(t) \leq 14.886 t^{5 / 2}
$$

Proof. Clearly,

$$
\begin{aligned}
& 0 \leq \mathcal{C}=\int_{0}^{1} t^{5 / 2} d A(t)=1-\left[\int_{1 / 2}^{3 / 4} \frac{3}{2} d t^{5 / 2}+\int_{3 / 4}^{1} d t^{5 / 2}\right] \approx 0.0217<1, \\
& G(t, s)= \begin{cases}G_{1}(t, s)=\frac{t^{3 / 2}(1-s)^{3 / 2}}{\Gamma(5 / 2)}, & 0 \leq t \leq s \leq 1 \\
G_{2}(t, s)=\frac{t^{3 / 2}(1-s)^{3 / 2}-(t-s)^{3 / 2}}{\Gamma(5 / 2)}, & 0 \leq s \leq t \leq 1 .\end{cases}
\end{aligned}
$$

Thus,

$$
\begin{aligned}
& \mathcal{G}_{A}(s)= \begin{cases}\frac{3}{2} G_{2}\left(\frac{1}{2}, s\right)-\frac{1}{2} G_{2}\left(\frac{3}{4}, s\right), & 0 \leq s<\frac{1}{2} \\
\frac{3}{2} G_{1}\left(\frac{1}{2}, s\right)-\frac{1}{2} G_{2}\left(\frac{3}{4}, s\right), & \frac{1}{2} \leq s<\frac{3}{4} \\
\frac{3}{2} G_{1}\left(\frac{1}{2}, s\right)-\frac{1}{2} G_{1}\left(\frac{3}{4}, s\right), & \frac{3}{4} \leq s \leq 1\end{cases} \\
& = \begin{cases}\frac{((3 / 2) \sqrt{2}-(3 / 4) \sqrt{3})(1-s)^{3 / 2}+2((3 / 4)-s)^{3 / 2}-6((1 / 2)-s)^{3 / 2}}{3 \sqrt{\pi}}, & 0 \leq s<\frac{1}{2}, \\
\frac{((3 / 2) \sqrt{2}-(3 / 4) \sqrt{3})(1-s)^{3 / 2}+2((3 / 4)-s)^{3 / 2}}{3 \sqrt{\pi}}, & \frac{1}{2} \leq s<\frac{3}{4} \\
\frac{((3 / 2) \sqrt{2}-(3 / 4) \sqrt{3})(1-s)^{3 / 2}}{3 \sqrt{\pi}}, & \frac{3}{4} \leq s \leq 1,\end{cases} \\
& \mathscr{H}(s)=\frac{(1-s)^{3 / 2}}{\Gamma(5 / 2)}+\frac{\mathcal{G}_{A}(s)}{0.9783} .
\end{aligned}
$$

Clearly, (H0) and (H1) hold. 
On the other hand, for any $\lambda_{i}>0, i=0,1, f\left(t, \lambda_{0} t, \lambda_{1}\right)=\lambda_{0}^{-1 / 8} t^{-3 / 8}+\lambda_{1}^{-1 / 3} t^{-1 / 4} \not \equiv 0$, $t \in(0,1)$, and

$$
\begin{gathered}
\kappa_{0}(s)=\frac{2}{5} s^{5 / 2}, \quad \kappa_{1}(s)=s^{3 / 2}, \\
0<\int_{0}^{1} \mathscr{H}(s) f\left(s, \lambda_{0} \kappa_{0}(s), \lambda_{1} \kappa_{1}(s)\right) d s \\
=10 \int_{0}^{1}\left[\frac{(1-s)^{3 / 2}}{\Gamma(5 / 2)}+\frac{\mathcal{G}_{A}(s)}{0.9783}\right]\left[\left(\frac{2}{5} \lambda_{0}\right)^{-1 / 8} s^{-9 / 16}+\lambda_{1}^{-1 / 3} s^{-3 / 4}\right] d s<+\infty .
\end{gathered}
$$

Thus (H2) is satisfied.

Now we compute $L, l$. Since

$$
\begin{array}{rl}
\int_{0}^{1} \mathcal{G}_{A}(s) f\left(s, \kappa_{0}(s), \kappa_{1}(s)\right) d s=10 \int_{0}^{1} \mathcal{G}_{A}(s)\left[\left(\frac{2}{5}\right)^{-1 / 8} s^{-9 / 16}+s^{-3 / 4}\right] d s & \\
= & 10 \int_{0}^{1 / 2}\left[\frac{((3 / 2) \sqrt{2}-(3 / 4) \sqrt{3})(1-s)^{3 / 2}+2((3 / 4)-s)^{3 / 2}-6((1 / 2)-s)^{3 / 2}}{3 \sqrt{\pi}}\right] \\
& \times\left[\left(\frac{2}{5}\right)^{-1 / 8} s^{-9 / 16}+s^{-3 / 4}\right] d s \\
+ & 10 \int_{1 / 2}^{3 / 4} \frac{((3 / 2) \sqrt{2}-(3 / 4) \sqrt{3})(1-s)^{3 / 2}+2((3 / 4)-s)^{3 / 2}}{3 \sqrt{\pi}}\left[\left(\frac{2}{5}\right)^{-1 / 8} s^{-9 / 16}+s^{-3 / 4}\right] \\
+ & 10 \int_{3 / 4}^{1} \frac{((3 / 2) \sqrt{2}-(3 / 4) \sqrt{3})(1-s)^{3 / 2}\left[\left(\frac{2}{5}\right)^{-1 / 8} s^{-9 / 16}+s^{-3 / 4}\right] d s=2.600,}{3 \sqrt{\pi}} \int_{0}^{1} \mathscr{H}(s)\left[\left(\frac{2}{5}\right)^{-1 / 8} s^{-9 / 16}+s^{-3 / 4}\right] d s \\
\int_{0}^{1} \mathscr{H}(s) f\left(s, \kappa_{0}(s), \kappa_{1}(s)\right) d s=10 & 10 \int_{0}^{1}\left[\frac{(1-s)^{3 / 2}}{\Gamma(5 / 2)}+\frac{\mathcal{G}_{A}(s)}{0.9783}\right]\left[\left(\frac{2}{5}\right)^{-1 / 8} s^{-9 / 16}+s^{-3 / 4}\right] d s=37.215 .
\end{array}
$$

Thus

$$
\begin{gathered}
L=\max \left\{1, \int_{0}^{1} \mathscr{H}(s) f\left(s, \kappa_{0}(s), \kappa_{1}(s)\right) d s\right\}=\max \{1,37.215\}=37.215 \\
l=\min \left\{1, \frac{1}{1-\mathcal{C}} \int_{0}^{1} \mathcal{G}_{A}(s) f\left(s, \kappa_{0}(s), \kappa_{1}(s)\right) d s\right\}=\min \{1,2.658\}=2.658
\end{gathered}
$$


So

$$
\begin{aligned}
\int_{0}^{1} \mathcal{G}_{A}(s) f\left(s, \frac{L}{l} \kappa_{0}(s), \frac{L}{l} \kappa_{1}(s)\right) d s & =10 \int_{0}^{1} \mathcal{G}_{A}(s)\left[\left(14 \times \frac{2}{5}\right)^{-1 / 8} s^{-9 / 16}+14^{-1 / 3} s^{-3 / 4}\right] d s \\
& =4.540 \geq 1-\mathcal{C}=0.9783,
\end{aligned}
$$

which implies that (3.16) holds. Then the BVP (1.4) has at least a positive solution $x(t)$, and there exist two constants

$$
\mathfrak{B}_{1}=\frac{\Gamma(\alpha-n+2)}{\Gamma(\alpha)}=\frac{2}{5}, \quad \boldsymbol{B}_{2}=L \boldsymbol{B}_{1}=14.886
$$

such that

$$
\frac{2}{5} t^{5 / 2} \leq x(t) \leq 14.886 t^{5 / 2}
$$

\section{Acknowledgment}

This work is supported by the National Natural Science Foundation of China (61201143) and the Fundamental Research Funds for the Central Universities (Grant no. HIT. NSRIF. 2010091), the National Science Foundation for Postdoctoral Scientists of China (Grant no. 2012M510956), and the Postdoctoral Funds of Heilongjiang Province (Grant no. LBHZ11128).

\section{References}

[1] T. T. Hartley, C. F. Lorenzo, and H. K. Qammer, “Chaos in a fractional order Chua's system," IEEE Transactions on Circuits and Systems I, vol. 42, no. 8, pp. 485-490, 1995.

[2] S. M. Caputo, "Free modes splitting and alterations of electrochemically polarizable media," Rendiconti Lincei, vol. 4, no. 2, pp. 89-98, 1993.

[3] T. J. Anastasio, "The fractional-order dynamics of brainstem vestibulo-oculomotor neurons," Biological Cybernetics, vol. 72, no. 1, pp. 69-79, 1994.

[4] R. A. Khan, "The generalized method of quasilinearization and nonlinear boundary value problems with integral boundary conditions," Electronic Journal of Qualitative Theory of Differential Equations, vol. 19, pp. 1-15, 2003.

[5] J. M. Gallardo, "Second-order differential operators with integral boundary conditions and generation of analytic semigroups," The Rocky Mountain Journal of Mathematics, vol. 30, no. 4, pp. 1265-1291, 2000.

[6] G. L. Karakostas and P. Ch. Tsamatos, "Multiple positive solutions of some Fredholm integral equations arisen from nonlocal boundary-value problems," Electronic Journal of Differential Equations, vol. 30, pp. 1-17, 2002.

[7] B. Ahmad, S. K. Ntouyas, and A. Alsaedi, "New existence results for nonlinear fractional differential equations with three-point integral boundary conditions," Advances in Difference Equations, vol. 2011, Article ID 107384, 11 pages, 2011.

[8] M. Feng, X. Liu, and H. Feng, "The existence of positive solution to a nonlinear fractional differential equation with integral boundary conditions," Advances in Difference Equations, vol. 2011, Article ID 546038, 14 pages, 2011.

[9] C. Corduneanu, Integral Equations and Applications, Cambridge University Press, Cambridge, UK, 1991. 
[10] R. P. Agarwal and D. O’Regan, Infinite Interval Problems for Differential, Difference and Integral Equations, Kluwer Academic Publishers, Dordrecht, The Netherlands, 2001.

[11] R. Ma and N. Castaneda, "Existence of solutions of nonlinear $m$-point boundary-value problems," Journal of Mathematical Analysis and Applications, vol. 256, no. 2, pp. 556-567, 2001.

[12] P. W. Eloe and B. Ahmad, "Positive solutions of a nonlinear $n$th order boundary value problem with nonlocal conditions," Applied Mathematics Letters, vol. 18, no. 5, pp. 521-527, 2005.

[13] X. Hao, L. Liu, Y. Wu, and Q. Sun, "Positive solutions for nonlinear $n$ th-order singular eigenvalue problem with nonlocal conditions," Nonlinear Analysis. Theory, Methods \& Applications A, vol. 73, no. 6, pp. 1653-1662, 2010.

[14] C. Yuan, "Multiple positive solutions for $(n-1,1)$-type semipositone conjugate boundary value problems of nonlinear fractional differential equations," Electronic Journal of Qualitative Theory of Differential Equations, no. 36, pp. 1-12, 2010.

[15] S. Zhang, "Positive solutions to singular boundary value problem for nonlinear fractional differential equation," Computers \& Mathematics with Applications, vol. 59, no. 3, pp. 1300-1309, 2010.

[16] X. Zhang and Y. Han, "Existence and uniqueness of positive solutions for higher order nonlocal fractional differential equations," Applied Mathematics Letters, vol. 25, no. 3, pp. 555-560, 2012.

[17] X. Zhang, L. Liu, and Y. Wu, "The eigenvalue problem for a singular higher order fractional differential equation involving fractional derivatives," Applied Mathematics and Computation, vol. 218, no. 17, pp. 8526-8536, 2012.

[18] X. Zhang, L. Liu, B. Wiwatanapataphee, and Y. Wu, "Positive solutions of eigenvalue problems for a class of fractional differential equations with derivatives," Abstract and Applied Analysis, vol. 2012, Article ID 512127, 16 pages, 2012.

[19] X. Zhang, L. Liu, and Y. Wu, "Multiple positive solutions of a singular fractional differential equation with negatively perturbed term," Mathematical and Computer Modelling, vol. 55, no. 3-4, pp. 1263-1274, 2012.

[20] X. Zhang, L. Liu, and Y. Wu, "Existence results for multiple positive solutions of nonlinear higher order perturbed fractional differential equations with derivatives," Applied Mathematics and Computation. In press.

[21] K. S. Miller and B. Ross, An Introduction to the Fractional Calculus and Fractional Differential Equations, John Wiley \& Sons, New York, Ny, USA, 1993.

[22] I. Podlubny, Fractional Differential Equations, vol. 198 of Mathematics in Science and Engineering, Academic Press, New York, NY, USA, 1999.

[23] J. R. L. Webb and G. Infante, "Non-local boundary value problems of arbitrary order," Journal of the London Mathematical Society, vol. 79, no. 1, pp. 238-258, 2009. 


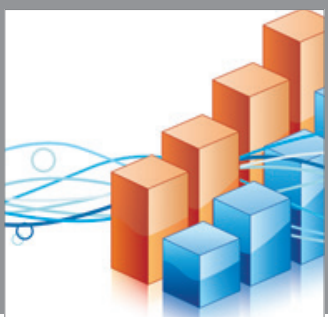

Advances in

Operations Research

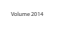

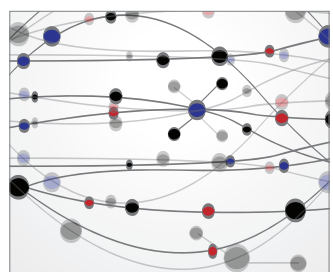

\section{The Scientific} World Journal
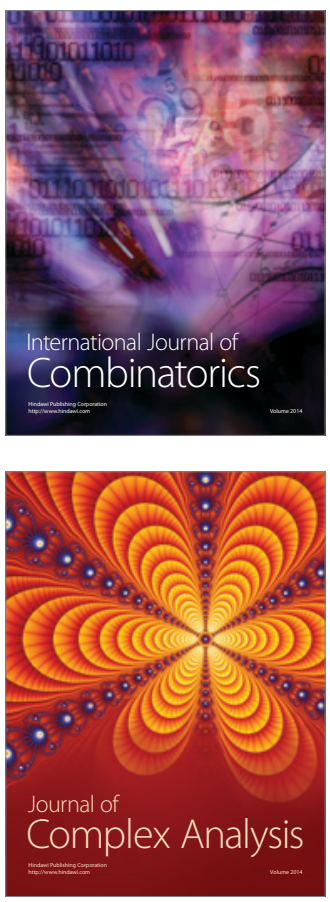

International Journal of

Mathematics and

Mathematical

Sciences
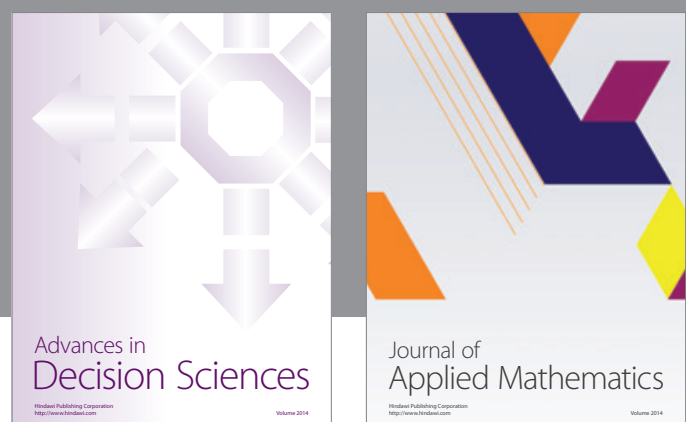

Journal of

Applied Mathematics
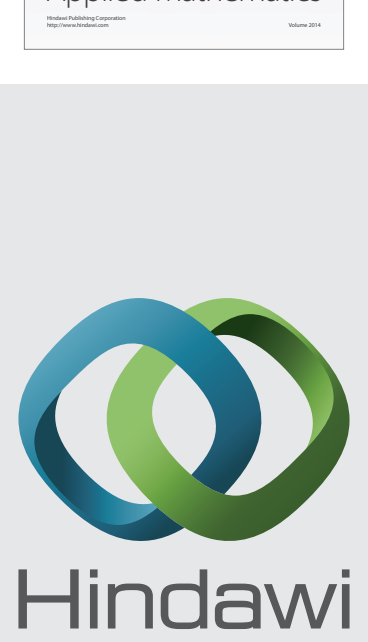

Submit your manuscripts at http://www.hindawi.com
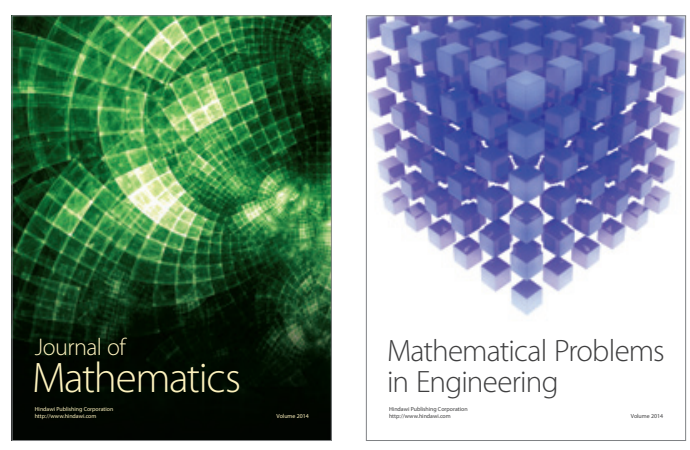

Mathematical Problems in Engineering
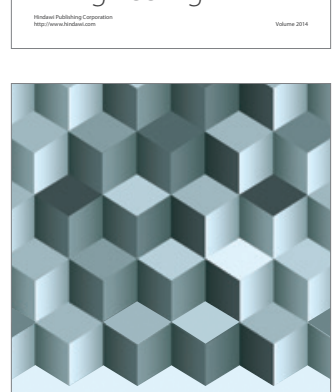

Journal of

Function Spaces
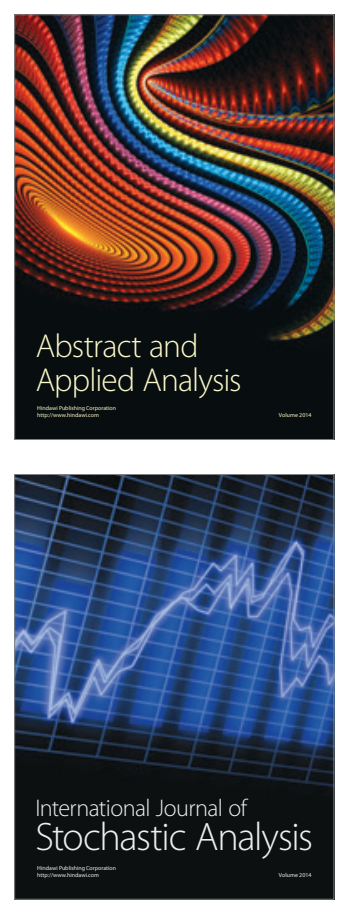

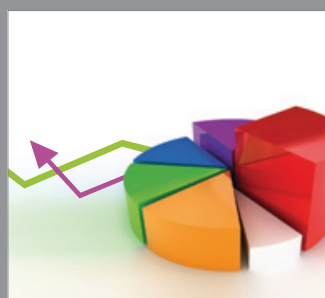

ournal of

Probability and Statistics

Promensencen
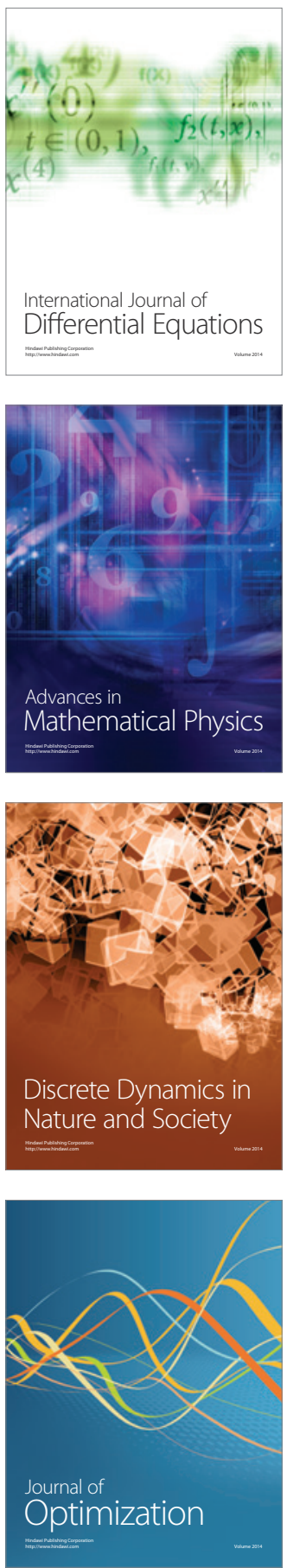